\title{
Umfassende Integrationsformel
}

\author{
Wie können Produkte oder Verfahren vergleichend bewertet werden? Diese \\ Frage stellt sich spätestens dann, wenn ökologische und betriebswirtschaftliche \\ Bewertung auseinander fallen. Im Folgenden wird eine umfassende Bewer- \\ tungsmethode entwickelt und am Beispiel von Verfahren zur Verwertung von \\ Bioabfällen illustriert. Im Kern wird hierzu vorgeschlagen, die unterschiedlichen \\ ökologischen Wirkungen über die Berechnung von Vermeidungskosten mit den \\ Betriebswirtschaftlichen Kostenwerten vergleichbar zu machen.
}

M Von Michael Steinfeldt it der Methode der Ökobilanzierung können die einzelnen Umweltauswirkungen der betrachteten Szenarien sehr detailliert ermittelt werden. Die betriebswirtschaftliche Betrachtung von Szenarien berïcksichtigt hingegen lediglich die Kosten und Erträge, die für das Unternehmen unmittelbar anfallen. Beim Vergleich von Systemen tritt oft der Fall ein, dass einzelne Systeme ökologischer, andere aber preiswerter sind. Bei derartigen Ergebnissen eröffnet sich somit ein Spannungsverhältnis zwischen der betriebswirtschaftlichen und der ökobilanziellen Analyse. Die zentrale Frage ist dann, inwieweit die zusätzlichen finanziellen Aufwendungen durch die verringerten Umweltbelastungen der aufwändigeren Verfahren zu rechtfertigen sind.

Diese Frage ist allerdings nicht mehr allein im Rahmen eines Vergleichs der betrachteten Systeme zu beantworten. Aus volkswirtschaftlicher Sicht ist nun zu diskutieren, inwiefern die kostenseitige Berücksichtigung von externen ökologischen Effekten die Szenarienbewertungen beeinflusst bzw. eine zusammengefasste Bewertung ermöglichen könnte.

\section{Ein umfassender Bewertungsansatz}

Zur Lösung dieser Problematik wird im Folgenden ein methodischer Ansatz vorgeschlagen, der eine solche umfassende ökonomische Bewertung von Systemvergleichen ermöglicht. Dieser besteht darin, dass für den Vergleich zweier Systeme

- einerseits die jeweiligen betriebswirtschaftlichen Kosten (in der folgenden Gleichung als Systemkosten SK1 und SK2 dargestellt) ermittelt und - andererseits die externen ökologischen Effekte der Differenz zwischen beiden Systemen mit Hilfe des Vermeidungskostenansatzes monetarisiert (in der folgenden Gleichung als Kostenwert Kex dargestellt) berücksichtigt werden.

Für den Vergleich zweier Systeme ergibt sich folgende Gleichung, wobei für das Ergebnis der Wert Ksv - Systemvergleichskostenwert - eingeführt wird:

$\mathrm{Ksv}_{\mathrm{sv}}=\mathrm{SK} 1-\mathrm{SK} 2-\mathrm{Kex}$.

Für die Ergebnisdiskussion des Systemsvergleiches unter Berücksichtigung der externen ökologischen Effekte ist in erster Linie das Vorzeichen des Systemvergleichskostenwertes Ksv und erst dann die Größe des Wertes maßgeblich. Wenn das ökologisch günstigere als erstes System und das kostengünstigere Verfahren als System 2 definiert wird, ergeben sich grundsätzlich zwei Möglichkeiten:

1. Bleibt der Systemvergleichskostenwert $>0$ bedeutet dies, dass System 2 sowohl rein betriebswirtschaftlich als auch unter zusätzlicher monetärer Berücksichtigung der externen ökologischen Nachteile gegenüber dem System 1 von Vorteil ist. Die ökologischen Vorteile des Systems 1 gleichen den betriebswirtschaftlichen Nachteil nicht aus. Aus volkswirtschaftlicher Sicht wäre es dann günstiger, System 2 zu präferieren und die eingesparten Mittel in effizientere Vermeidungstechnologien $\mathrm{zu}$ investieren.

2. Ist der Systemvergleichskostenwert dagegen $\leq \mathbf{0}$, dann bewirkt die Berücksichtigung der externen Effekte eine Verschiebung in der Systembewertung. Das System 1 ist im Vorteil, das heißt die zusätzliche monetäre Berücksichtigung der Vermeidungskosten des Systems 2, die zur Aufhebung der ökologischen Nachteile gegenüber System 1 notwendig wären, führt dazu, dass der rein betriebswirtschaftliche Nachteil mehr als ausgeglichen wird. Aus volkswirtschaftlicher Sicht wäre es dann günstiger, System 1 zu präferieren und die im Vergleich mit System 2 zusätzlichen Mittel zu investieren, da sie hier ökologisch am effizientesten eingesetzt werden.

\section{Berechnung der ökologischen externen Effekte}

Damit stellt sich die Frage nach der Bestimmung des Wertes Kex, also der Gesamtvermeidungskosten der Systemdifferenz. Zur Berechnung wurde die im Kasten dargestellte Formel entwickelt.

\section{Berechnungsformel}

$K_{e x}=\sum_{i}\left(W P 2_{i}-W P 1_{i}\right) \times$ ÄqEi $\times V_{K_{E}}+$ $\sum_{j}\left[\operatorname{Max}\left(\left(W P 2_{k}-W P 1_{k}\right) \times \ddot{A}_{q k} \times V_{K_{k}}\right)\right]$

Kex Kostenwert der der Differenz der externen ökologischen Effekte der Systeme 1 und 2 pro funktionaler Einheit

WP1/2 berechnetes Netto-Wirkungspotenzial pro funktionaler Einheit für das System $1 / 2$

E Leitemission, also die Emission, die im Systemvergleich zu wesentlichen Umweltauswirkungen führt und dementsprechend die Ökobilanzergebnisse bestimmt

Äq Äquivalenzfaktor zur Bestimmung des Beitrages von Leitemissionen zum Umweltwirkungspotenzial

VKE durchschnittliche Vermeidungskosten der Leitemission

Max Maximum

i Menge der Umweltwirkungskategorien, für die ein einfacher Zusammenhang zwischen einer Leitemission und einer Wirkungskategorie besteht

k Menge der Umweltwirkungskategorien, bei der ein Zusammenhang zwischen einer Leitemission und mehreren Wirkungskategorien besteht

Diese Berechnungsformel greift die Methodik der Wirkungsabschätzung im Rahmen der Ökobilanzierung auf und verknüpft diese mit dem Vermeidungskostenansatz, um die verschiedenen Umweltwirkungskategorien zu monetarisieren und letztlich die Systemdifferenz in Kostengrößen als Summenwert vergleichbar zu machen.

Der erste Teil der Gleichung bezieht sich auf die Umweltwirkungen, für die ein einfacher Zusammenhang zwischen einer Leitemission und einer Wirkungskategorie besteht. Als Beispiel hierfür seien Kohlendioxidemissionen genannt. Die in der Sachbilanzierung ermittelten KohlendioxidEmissionen besitzen allein ein Wirkungspotenzial für den Treibhauseffekt. Der Beitrag der Kohlendioxid-Emissionen zum Treibhauseffekt wird über den Wirkungsäquivalentfaktor $\ddot{q} q$ bestimmt. Dieser Wert beträgt im Beispielfall 1.

Aus der Ökobilanzierung sind die Werte WP2 und WP1 bekannt und somit auch der Differenzbetrag zwischen beiden. Vermeidungskostenansätze $V K_{E}$ sind bisher zumeist für einzelne Emis- 
sionen berechnet worden. Das Produkt aus Vermeidungskosten einer (Leit)Emission und ihrem Äquivalenzfaktor ergibt die Vermeidungskosten für die Wirkungskategorie. Diese vereinfachendeVerknüpfung ist aus meiner Sicht legitim, sofern es sich um Emissionen handelt, die die Ökobilanz wesentlich bestimmen. So werden Vermeidungskosten für Kohlendioxid häufig auch als Vermeidungskosten für den Treibhauseffekt angesehen. Als Ergebnis aus dem Produkt des Differenzbetrags (WP1-WP2) und dem Vermeidungskostenwert der Wirkungskategorie erhält man wirkungskategoriespezifische Vermeidungskosten für den Differenzbetrag. Der Summenwert wird über alle derartigen Wirkungskategorien gebildet.

Der zweite Teil der Gleichung berücksichtigt dagegen die Umweltwirkungen, für die ein $\mathrm{Zu}$ sammenhang zwischen einer Leitemission und mehreren Wirkungskategorien besteht. Als Illustration seien Ammoniak- und Cadmiumemissionen genannt. Ammoniakemissionen besitzen Wirkungspotenziale zur terrestrischen Eutrophierung, zur Versauerung sowie zum so genannten PM10-Risiko, das aus der Belastung von Feinstäuben entsteht. Cadmiumemissionen wirken sich dagegen negativ in den Wirkungskategorien Schadstoffeintrag in Boden sowie Krebsrisiko aus.

Derartige mehrfache Zuordnungen einer Leitemission zu mehreren Wirkungskategorien verkomplizieren die Rechnung mit Vermeidungskosten. Die verschiedenen wirkungskategoriespezifische Vermeidungskosten dürfen nicht aufsummiert werden, sondern es darf im Beispiel
Ammoniak aus den drei Werten nur der Maximalwert für die weitere Berechnung berïcksichtigt werden, da mit dem höchsten Vermeidungskostenwert auch die höchsten Minderungen in den anderen Umweltkategorien erzielt werden. Diese letztlich ,positiven Gutschriften“ sollen aber im Weiteren nicht berücksichtigt werden. Abschließend wird der Summenwert über alle derartigen Fälle j gebildet. Die Berechnung ist allerdings nur schlüssig, wenn jede Wirkungskategorie nur jeweils einmal in der Gleichung vorkommt. Sonst verstärkt sich die Problematik der „Gutschriften“ noch.

Die Summe aus beiden Gleichungsteilen ergibt dann den Wert $\mathrm{K}_{\text {ex }}$, also den Kostenwert der externen ökologischen Effekte der Differenz der Systeme 1 und 2. Dieser Wert stellt somit die Vermeidungskosten für die ökologische Differenz der beiden betrachteten Systeme dar und muss dem ökologisch schlechteren System zugerechnet werden.

\section{Bioabfallverwertungssysteme als Beispiel}

In dem von der Deutschen Bundesstiftung Umwelt (DBU) eingerichteten Förderschwerpunkt Bioabfallverwertung befasste sich ein Projekt mit der ökologisch-ökonomischen Bewertung der Verfahren der Bioabfallbehandlung. Dieses wurde als Kooperationsprojekt vom ifeu-Institut für Energie- und Umweltforschung Heidelberg, dem Institut für ökologische Wirtschaftsforschung (IÖW) sowie PlanCoTec bearbeitet (1). Das IÖW hatte insbesondere die Aufgabe, die vom ifeu-Institut vergleichend ökologisch bilanzierten Entsorgungsoptionen von biologisch-

Abb. 1: Vermeidungskosten einzelner Emissionen gegenöber dem Szenario KompGeschl_2

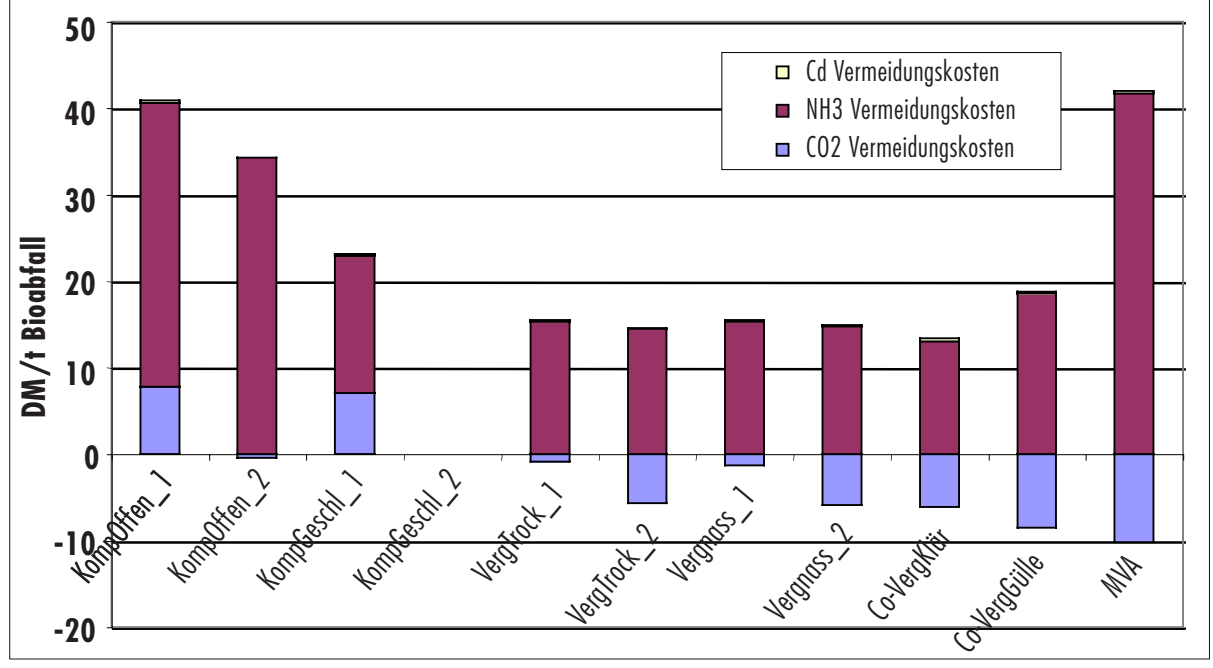

Quelle: eigene Darstellung organischen Abfällen ökonomisch zu bewerten sowie eine Beurteilung im volkswirtschaftlichen Kontext vorzunehmen. Für die betriebswirtschaftliche Bewertung wurden die Entsorgungsbzw. Verwertungsoptionen von Bioabfall aus Haushalten ausgewählt.

Die ökobilanzielle Bewertung der betrachteten Optionen kommt zu dem Ergebnis, dass neben der - vom IÖW betriebswirtschaftlich nicht untersuchten - Eigenkompostierung die geschlossene Kompostierung mit dem Behandlungsziel Fertigkompost zu bevorzugen ist. Außerdem kann festgestellt werden, dass keine betrachtete Entsorgungsalternative ökologisch derart schlecht abschneidet, dass sie unter ökologischen Gesichtspunkten ausgeschlossen werden sollte (2). Die betriebswirtschaftliche Analyse kommt hingegen zu unterschiedlichen, teilweise entgegengesetzten Ergebnissen (vgl. auch die spezifischen Verwertungskosten in Abb. 2). Hier schneiden die offene Kompostierung sowie die Co-Vergärungsszenarien mit spezifischen Verwertungskosten von 65 bis 95 Mark pro Tonne Bioabfall am besten ab (3).

\section{Ökonomie vs. Ökologie?}

Die ökologisch beste Variante, Containerkompostierung mit geschlossener Hallennachrotte (KompGeschl_2), ist die ökonomisch teuerste Variante mit Verwertungskosten von 174 Mark pro Tonne Bioabfall. Dies ist im Grundsatz nicht überraschend, da das Verfahren die technologisch aufwändigste Nachrottetechnologie erfordert, um zu einer relativ geringeren Umweltbelastung zu kommen. Die Berücksichtigung der volkswirtschaftlichen Sicht bei der ökonomischen Bewertung erfolgte entsprechend der vorgestellten Methodik durch die Berechnung von Vermeidungskosten der jeweiligen Systemdifferenzen bezogen auf die ökologisch beste Variante.

Als relevante Wirkungskategorien, denen eine sehr große ökologische Bedeutung in der Ökobilanzierung beigemessen wurde, sind einerseits der Treibhauseffekt sowie andererseits das Krebsrisiko (Humantoxizität) zu nennen. Eine große Bedeutung und gleichzeitig einen hohen spezifischen Beitrag besaßen in der ökologischen Bewertung die Wirkungskategorien Versauerung, terrestrische Eutrophierung, PM10-Risiko (Humantoxizität) sowie Cadmium-Eintrag Boden. Als Leitemissionen, die diese Wirkungskategorien wesentlich bestimmen, konnten Kohlendioxid, Cadmium und Ammoniak identifiziert werden. 
Abb. 2: Ökonomischer Systemvergleich unter Berücksichtigung der externen ökologischen Effekte

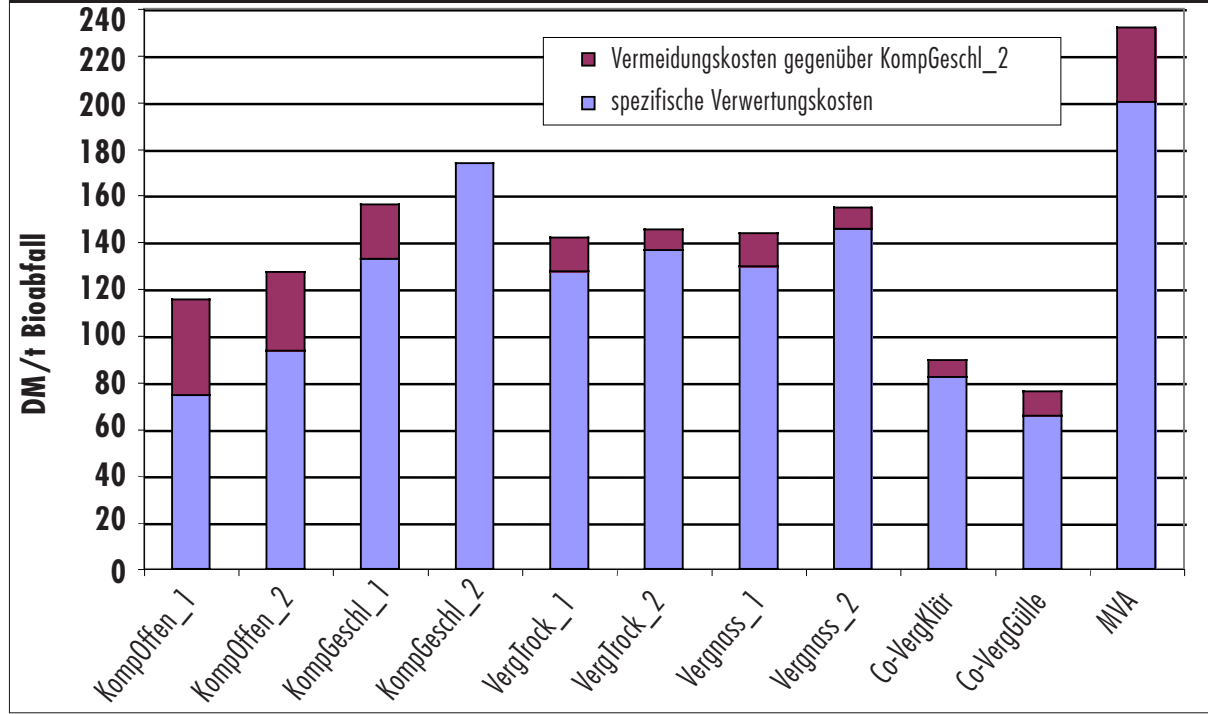

Quelle: eigene Darstellung

Aus diesem Grunde wurden diese Emissionen mit entsprechenden Vermeidungskosten in die Berechnung der Gesamtvermeidungskosten aufgenommen, und zwar mit Ausgangswerten von 100 Mark pro Tonne Kohlendioxid, 11.900 Mark pro Tonne Ammoniak und 3,5 Millionen Mark pro Tonne Cadmium (4).

Eine vergleichende Übersicht der ermittelten spezifischen Vermeidungskosten ist in Abb. 1 dargestellt. Für den Vergleich relevante Vermeidungskosten verursachen nur die beiden Emissionen Ammoniak und Kohlendioxid - in dieser Reihenfolge. Außerdem wird deutlich, dass insbesondere in den offenen Kompostierungsszenarien (KompOffen) sowie bei der Müllverbrennung (MVA) beträchtliche Investitionen getätigt werden müssten, um die jeweiligen ökologischen Nachteile gegenüber dem ökologisch besten Szenario KompGeschl_2 auszugleichen. In den (Co-)Vergärungsszenarien (CoVerg) heben sich teilweise Vermeidungskosten von Ammoniak und Kohlendioxid auf.

\section{Ökologisch-ökonomischer Gesamtvergleich}

Die berechneten Vermeidungskosten werden im nächsten Schritt den jeweiligen Szenarien auf die Verwertungskosten zugerechnet (s. Abb. 2). Dieser Summenwert ist nach der vorgestellten Methode direkt mit den Verwertungskosten des ökologisch besten Szenarios vergleichbar. Beim Vergleich der Ergebnisse stellt sich heraus, dass die jeweiligen zuzurechnenden Vermeidungskosten die grundsätzlichen Aussagen der betriebswirtschaftlichen Bewertung unterstïtzen, wobei eine Differenzierung bei den bisher besten Szenarien festzustellen

ist. Die spezifischen Vergleichskosten der (Co) Vergärungsverfahren erhöhen sich nur um 7 bis 15 Mark pro Tonne Bioabfall, wohingegen sich die offenen Kompostierungsverfahren um 34 bis 41 und auch die Müllverbrennung um 32 Mark pro Tonne Bioabfall verschlechtern.

Zusammengefasst kommt die ökologisch-ökonomische Bewertung zu dem Ergebnis, dass

- im Grundsatz die betrachteten Verfahren zur Verwertung von Bioabfällen der Entsorgung durch Müllverbrennung vorzuziehen sind,

- die ökonomisch kostengünstigeren Verfahren - insbesondere die Co-Vergärung mit Gülle vorzuziehen sind, da die mit den aufwändigeren Verfahren zu erreichenden zusätzlichen Umweltentlastungen mit hohen Vermeidungskosten verbunden sind.

Aus umweltpolitischer Sicht bedeutet dies, dass die aufwändigeren Verwertungsverfahren zwar durchaus positiv zu bewerten sind, dass sie aber im Rahmen einer Gesamtstrategie nicht die vorzuziehenden Lösungen sein können. Finanzielle Mittel für nationale Klimaschutz- oder Ammoniakminderungsstrategien könnten in anderen Bereichen der Volkswirtschaft effizienter eingesetzt werden.

Diese abstrakte Feststellung klammert natürlich erst einmal die vorhandenen institutionellen und rechtlichen Zuständigkeiten und damit verbundenen Restriktionen der staatlichen Institutionen für die Umsetzung effektiver Minderungsstrategien in den verschiedenen Politikfeldern aus. Beispielsweise stehen entfallende Gebühreneinnahmen im Abfallbereich der Kommune natürlich nicht automatisch für Förderprogramme zur Energieeinsparung bereit, sondern würden erst einmal direkt den Bürger finanziell entlasten.
Der entwickelte methodische Ansatz zur umfassenden ökonomischen Bewertung von ökologischen Systemvergleichen kann aber als Beitrag zur wissenschaftlichen Diskussion über die Effizienz von umweltpolitischen Maßnahmen gerade auch im ressortïbergreifenden und im volkswirtschaftlichen Kontext verstanden werden.

\section{Anmerkungen}

(1) Hierbei handelte es sich um das DBU-Forschungsprojekt "Untersuchungen zur Umweltverträglichkeit von Systemen zur Verwertung von biologisch-organischen Abfällen" (AZ 08848). Die Publikation des IÖW-Projektberichtes ist in der Veröffentlichungsreihe "Initiativen zum Umweltschutz" der DBU vorgesehen.

(2) Vgl. Vogt, R. et al.: Untersuchungen zur Umweltverträglichkeit von Systemen zur Verwertung von biologisch-organischen Abfällen. Teilbericht des ifeu-Institutes zum gleichnamigen DBU-Projekt, Heidelberg 2001.
(3) Für das Szenario Co-Vergärung Klärwerk muss dabei allerdings betont werden, dass es sich bei der Berechnung nur um eine Grenzkostenbetrachtung handelt, das heift hier wurden nur Zusatzinvestitionen berücksichtigt und freie Kapazitäten im vorhandenen Faulbehälter und der übrigen Infrastruktur unterstellt. Für eine ausführliche und erläuternde Darstellung der jeweiligen Berechnungsannahmen und -quellen muss aus Platzgründen auf die in Anm. 1 erwähnte Publikation verwiesen werden.

(4) Vgl. zu den Vermeidungskosten von Kohlendioxid z.B. Matthes, F.: Anmerkungen zum Energiebericht des BMWi „Nachhaltige Energiepolitik für eine zukunftsfähige Energieversorgung", Öko-Institut, Berlin 2002; zu Ammoniak FAL et al.: Anpassung der deutschen Methodik zur rechnerischen Emissionsermittlung an internationale Richtlinien sowie Erfassung und Prognose der Ammoniak-Emissionen der deutschen Landwirtschaft und Szenarien zu deren Minderung bis zum Jahre 2010, Abschlussbericht UBA-Projekt, Berlin 2001; zu Cadmium Klepper, G./ Michaelis, P./ Mahlau, G.: Industrial Metabolism: A Case Study of the Economics of Cadmium Control, Tübingen 1995.

\section{Der Autor}

Michael Steinfeldt ist wissenschaftlicher Mitarbeiter im Forschungsfeld Ökologische Unternehmenspolitik des Instituts für ökologische Wirtschaftsforschung. Kontakt: IÖW, Potsdamer Str. 105, 10785 Berlin. Tel. 030/ 884594-18, Fax 030/ 8825439, E-mail: michael.steinfeldt@ioew.de 
(c) 20I0 Authors; licensee IÖW and oekom verlag. This is an article distributed under the terms of the Creative Commons Attribution Non-Commercial No Derivates License (http://creativecommons.org/licenses/by-nc-nd/3.o/), which permits unrestricted use, distribution, and reproduction in any medium, provided the original work is properly cited. 